\section{Betulsion regeneriert die Hautbarriere}

E ine geschädigte Hautbarriere erleichtert das Eindringen von Kontaktallergenen und Irritanzien in die Haut. Emulgatoren in herkömmlichen Hautpflegemitteln können die Barrierefunktion der Haut stören, indem sie die natürlichen Hautlipide der Hornschicht emulgieren und die Lipidlamellen der Oberhaut öffnen. Beim Waschen unterstützen sie die Entfettung der Haut, in deren Folge die eingelagerte Feuchtigkeit schneller verdunstet.

Dass stabile Emulsionen auch ohne Emulgatoren auskommen können, zeigt das Beispiel der „Betulsion“ in Betulinhaltigen Hautpflegeprodukten wie Imlan ${ }^{\circledR}$. Das Triterpen Betulin ist Hauptbestandteil eines Extrakts aus weißem Birkenkork, der antientzündliche, antibakterielle, juckreizlindernde und wundheilungsfördernde Eigenschaften hat. Birkenkork sei das triterpenreichste Gewebe der Pflanzenwelt, erklärte Prof. Dr. Stefan Martin, Freiburg.

Betulin stabilisiert Emulsionen zu einer Wasser-in-Öl-Emulsion (PickeringEmulsion), ohne dass weitere Inhalts- stoffe wie Emulgatoren oder Konservierungsmittel erforderlich sind. So kann empfindliche Haut mit nur drei Inhaltsstoffen (Betulin, Wasser, Jojobaöl) gepflegt werden, auf potenziell allergene Inhaltsstoffe und Irritanzien kann verzichtet werden.

Wie Prof. Dr. Wolfgang Gehring, Karlsruhe, in experimentellen Untersuchungen festgestellt hat, ist die mit Betulsion behandelte Haut vor dem schäd-

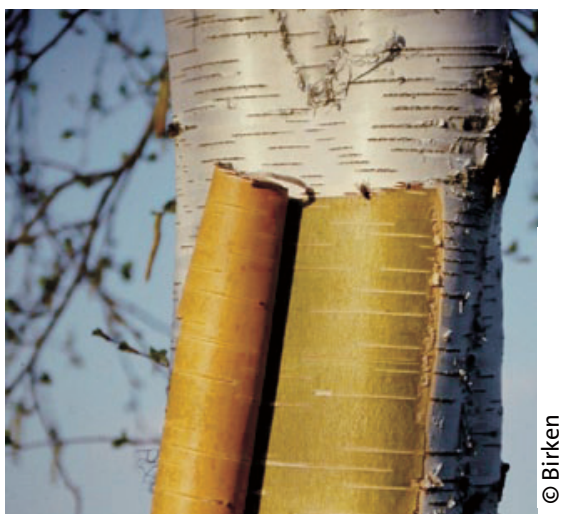

Aus dem Kork der Birkenrinde wird Betulin gewonnen. lichen Einfluss von waschaktiven Substanzen geschützt. Nach siebentägiger Behandlung mit Betulsion Pur oder mit Betulsion Plus - sie enthält zusätzlich Harnstoff und Bienenwachs - kam es, anders als bei unbehandelter Haut, nach dem Waschen mit Natriumlaurylsulfat nicht zu einer Erhöhung des transepidermalen Wasserverlusts.

An Haut mit gestörter epidermaler Barrierefunktion nach wiederholten Waschungen mit Natriumlaurylsulfat zeigten sich die regenerativen Eigenschaften der Betulsion: Im Vergleich zur unbehandelten Haut verbesserte sich die Hornschichtfeuchtigkeit signifikant (Pur: $\mathrm{p}<0,01$; Plus: $\mathrm{p}<0,001$ ) und der transepidermale Wasserverlust nahm als Zeichen einer Barrierestabilisation $a b$ (je $\mathrm{p}<0,001$ ). Darüber hinaus gingen auch entzündliche Begleitreaktionen signifikant zurück (Laszczyk MN. Aktuelle Dermatologie 2009; 35: 1-5).

Dagmar Jäger-Becker

Symposium „Die Betulin-Emulsion - hypoallergene Pflege aus der Birkenrinde" im Rahmen des 6. Deutschen Allergiekongresses. Wiesbaden, 10. September 2011. Veranstalter: Birken, Niefern-Öschelbronn

\title{
Angioödeme interdisziplinär betreuen
}

A ngioödeme sind flüchtige Ödeme, die in unregelmäßigen Zeitabständen auftreten. Sie können die Haut, seltener aber auch Zunge, Glottis, Larynx und den Magen-Darm-Trakt betreffen. „Oberstes Ziel bei der Betreuung und Behandlung von Patienten mit einem Angioödem muss es sein, eine Erstickung zu vermeiden", betonte Prof. Dr. Konrad Bork, Mainz. Rezidivierende Angioödeme können auf einem genetisch bedingten oder erworbenen Mangel an funktionsfähigem C1-Esterase-Inhibitor (C1-INH) beruhen. Der C1-INHMangel aktiviert indirekt das Kinin-System, was die Neigung zur Ödembildung fördert. Der Hauptmediator des hereditären Angioödems ist Bradykinin. Angioödeme können aber auch durch Hemmer des Angiotensinkonversionsenzyms (ACE) und nichtsteroidale Antirheuma- tika ausgelöst werden oder im Zusammenhang mit einer Urtikaria auftreten.

Angioödeme im Kopf-Hals-Bereich stellen häufig eine Notfallsituation dar und erfordern eine schnelle Intervention. Ein akutes Ödem der oberen Atem-Schluck-Straße bedarf einer interdisziplinären Betreuung durch HNOArzt, Anästhesisten und gegebenenfalls Pädiater, erläuterte PD Dr. Murat Bas, München.

Für die Therapieentscheidung ist die Differenzierung von allergischem und nicht allergischem Ödem wichtig. Bei allergischen Ödemen helfen laut Bas Antihistaminika, Glukokortikoide und gegebenenfalls Adrenalin. Nicht allergische Ödeme sind in den meisten Fällen entzündungsbedingt oder durch ACEHemmer induziert. ACE-Hemmer-induzierte Angioödeme treten überwie- gend im Kopf-Hals-Bereich auf - oftmals erst Jahre nach Therapiebeginn.

Noch gibt es keine zugelassene medikamentöse Therapie für diese Form des Angioödems. Erste Maßnahme ist das Absetzen des ACE-Hemmers. Bas berichtete, der Bradykinin-B2-Rezeptorantagonist Icatibant $\left(\right.$ Firazyr $\left.^{\circledR}\right)$, der für die Therapie des hereditären Angioödems zugelassen ist, habe sich in einer offenen Studie auch beim ACE-Hemmer-induzierten Angioödem bewährt. Zudem sei Icatibant kürzlich in einer Doppelblindstudie bei Patienten mit ACE-Hemmer-induzierten Angiödemen mit Kortison verglichen worden. Die Ergebnisse dieser Studie werden in Kürze erwartet.

Abdol A. Ameri

Klinisch-wissenschaftliches Symposium

„Angioödem“ im Rahmen des 6. Deut-

schen Allergiekongresses. Wiesbaden,

9. September 2011. Veranstalter: Shire, Köln 